\title{
New glucose metrics do not associate with carotid artery wall thickness and endothelial function in type 1 Diabetes
}

\section{Antonio Cutruzzolà}

Universita degli Studi Magna Graecia di Catanzaro Dipartimento di Medicina Sperimentale e Clinica

\section{Concetta Irace}

Universita degli Studi Magna Graecia di Catanzaro Dipartimento di Scienze Mediche

https://orcid.org/0000-0001-5182-5473

\section{Martina Parise}

Universita degli Studi Magna Graecia di Catanzaro Dipartimento di Medicina Sperimentale e Clinica

\section{Raffaella Fiorentino}

Azienda Ospedaliero Universitaria Materdomini

\section{Milena Barone}

Universita degli Studi Magna Graecia di Catanzaro Dipartimento di Medicina Sperimentale e Clinica

Agostino Gnasso ( $\sim$ gnasso@unicz.it )

https://orcid.org/0000-0002-9220-9754

\section{Original investigation}

Keywords: Glucose metrics, time in range, endothelial dysfunction, flow-mediated dilation, intima-plus media thickness, type 1 diabetes

Posted Date: May 20th, 2020

DOI: https://doi.org/10.21203/rs.3.rs-27649/v1

License: (a) (1) This work is licensed under a Creative Commons Attribution 4.0 International License. Read Full License 


\section{Abstract \\ Background}

Patients with Type 1 diabetes (T1D) have increased cardiovascular morbidity and mortality.

Atherosclerosis is a progressive disease and can be detected non-invasively in the pre-clinical stage by measuring intima-media thickness (IMT) and flow mediated dilation (FMD). Glycated hemoglobin ( $\mathrm{HbA1c}$ ) does not associate with IMT and FMD. We have designed the present study to evaluate whether time spent in the target range, below the target range, and glycemic variability associate with common carotid artery IMT and FMD in T1D.

\section{Methods}

78 T1D patients and 35 healthy control subjects were enrolled and underwent measurement of IMT and evaluation of FMD after ischemia and during 6 min of hand-grip exercise.

\section{Results}

Mean age of participants was $34 \pm 13$ years; mean disease duration and HbA1c were $17 \pm 9$ years, and $7.7 \pm 0.9 \%(61 \mathrm{mmol} / \mathrm{mol})$. IMT was significantly higher in T1D compared with controls $(643 \pm 196 \mathrm{vs}$. $536 \pm 111 \mu ; p<0.001)$ and FMD after ischemia significantly lower $(7.5 \pm 4.1$ vs. $10.1 \pm 1.9 \% ; p<0.0001)$. FMD evaluated during exercise every min up to 6 min was also significantly lower in T1D $(p<0.0001)$. No significant relationship between IMT, FMD, and glucose metrics was found.

\section{Conclusions}

The present findings confirm that young adult patients with T1D have clinically relevant early vascular abnormalities. The absence of relationships with the glucose metric underlines the complexity of the interplay between atherosclerosis and diabetes. It stimulates the search for new parameters or new cutoffs better associated with the initial stages of the disease.

\section{Background}

Type 1 diabetes (T1D) is a well-established risk factor for cardiovascular disease (CVD), which still represent the major causes of mortality in this group of patients. The risk for cardiovascular mortality is up to six-eight times higher in T1D than in subjects without diabetes, and even two times higher than in patients with type 2 diabetes $(1,2)$. Patients with T1D in the first decade of life have a risk of developing CVD dramatically higher compared to patients developing T1D after the second and third decades of life (3). The high risk of CVD in diabetic patients is attributable to several mechanisms, some of which are linked to chronic hyperglycemia. Diabetes promotes the imbalance between atheroprotective and 
atherogenic properties of the endothelium reducing bioavailability of nitric oxide (NO), which is the principal regulator of vascular tone and the major inhibitor of vasoconstrictors and pro-inflammatory molecules (4). Currently, atherosclerosis can be detected early in the preclinical stage. Markers of early atherosclerosis and predictors of incident cardiovascular events are the thickening of the arterial wall and the dysfunction of the endothelium. The ultrasound-based technique offers the opportunity to detect these morphological and functional abnormalities, increasing the chance to individualize patients requiring intensive treatment (5-9). Several papers have described the association between diabetes, arterial wall thickening and endothelial dysfunction (10-15). It is interesting that preclinical atherosclerosis in patients with diabetes correlates with age, disease duration, systolic blood pressure but not with $\operatorname{HbA} 1 \mathrm{c}(11,12,16)$. HbA1c is the gold standard for monitoring glycemia and predicting overt micro- and macro-angiopathy. However, it does not reflect the extent of glycemic excursion, the burden of hypoglycemia, and the overall time spent in the target range in a specific time interval. This information can be obtained by analyzing grouped daily blood glucose measurements. The recent introduction of diabetes management software has enhanced the automated analysis of glucose data collected by selfmonitoring blood glucose (SMBG) and continuous glucose monitoring system (CGM). In detail, the systems allow us to create and implement the use of new glucose metrics such as the percentage of time spent in the target range (TIR), below the target range (TBR), above the target range (TAR), and glycemic variability (GV) (17).

Two recent papers have described a close relationship between TIR and glycated hemoglobin (HbA1c) using different data-sets from observational studies and randomized controlled trials $(18,19)$. On average, a TIR of $70 \%$ corresponds to $\mathrm{HbA} 1 \mathrm{c}$ of $7 \%$. Unfortunately, prospective studies demonstrating the predictive role of TIR on diabetes complications are lacking. Two retrospective studies and one crosssectional study have demonstrated a significant and inverse relationship between TIR and the presence of microangiopathy, specifically retinopathy and autonomic neuropathy (20-22). The results of these studies have prompted us to investigate the association between TIR, TBR, TAR, and GV and subclinical atherosclerosis detected by ultrasound in T1D patients. The hypothesis is that the overall time spent in the target range, the burden of hypoglycemia, and the excursion of blood glucose rather than $\mathrm{HbA1c}$ might associate with carotid wall thickening and brachial artery endothelial dysfunction.

\section{Methods}

Study design and Patients: This is an observational study including outpatients with T1D who were monitoring glucose by CGM or by meters uploading glucose data into the Accu-Chek Connect diabetes management system (DMS). Exclusion criteria were: known cardiovascular disease (myocardial infarction, angina, stroke, TIA, peripheral arterial disease), use of vasoactive drugs as calcium channel blocker, nitrates, beta and alfa blockers, use of hybrid closed-loop system or (predictive) low glucose suspend, concomitance of diseases such as infection, surgery, and any acute event requiring intensification of insulin treatment, treatment with corticosteroids, and pregnancy. The research was approved by the local Ethical Committee, and only patients who gave their informed consent were enrolled in the study. Fasting plasma glucose (FPG) and HbA1c were measured at the time of the 
recruitment. HbA1c was measured by high-performance liquid chromatography aligned with DCCT (HbA1c-DCCT); FPG was measured by commercially available kits. Disease duration and ongoing treatment were obtained from clinical files. As controls, age, and sex, comparable healthy subjects without diabetes were recruited.

Novel glucose parameters TIR, TAR, TBR, mean daily glucose, and standard deviation were collected 2 weeks and 3 months before the vascular study by DMS uploading and analyzing CGM glucose data. The most recent 2 weeks are a sufficient collection interval evaluating the overall time spent in the target range. A more extended collection period offers a more robust evaluation of time spent in hypoglycemia and glycemic variability (17).

Vascular study. The study was performed in the morning using an echo-Doppler Philips HD 11XE (Royal Philips Electronics, the Netherlands) equipped with a 12-3 MHz linear array transducer and simultaneous ECG recording, in a temperature-controlled $\left(20-24^{\circ} \mathrm{C}\right)$ room, and after the patient was at rest for 10 minutes in the supine position. Participants were asked to abstain from exercise, alcohol, caffeine, food, and smoking for $12 \mathrm{~h}$ before the study.

Participants were invited to extend the neck to visualize the common carotid artery (CCA) $1 \mathrm{~cm}$ below the carotid bulb in three different projections, anterior, lateral, and posterior. The transducer position was then adjusted to obtain an angle between the ultrasound beam and the longitudinal axis of the vessel at $90^{\circ}$. The gain was fine-tuned to improve the image of the intima plus media complex of the far wall defined as the distance between the leading edge of the lumen-intima interface and the inner edge of the mediaadventitia interface. Images were then recorded for the offline measurement of intima-media thickness (IMT) using a dedicated software (Autodesk ${ }^{\circledR}$ Design Review, BSA Italy) (23).

IMT was measured at the right and left CCA as the mean of the three projections. The maximal value between those measured at the right and left CCA was used for statistical analyses and defined as mean maximal IMT of CCA.

Eleven participants ( 6 with T1D and 5 healthy subjects) were studied twice apart to estimate the intraoperator reproducibility of the IMT measurement expressed as the coefficient of correlation between two measurements (0.98).

The endothelial function of the brachial artery was evaluated in the non-dominant arm by two different tests using ischemia and exercise as stimuli $(24,25)$. Both stimuli cause a reduction of peripheral resistance downstream to the brachial artery that, in turn, causes an increase of brachial artery blood flow velocity. This phenomenon, defined as reactive hyperemia, is responsible for the following brachial artery dilation known as flow-mediated dilation (FMD). The magnitude of FMD is proportional to the ability of the endothelium to dilate in response to stimuli. FMD was expressed as the percentage change of brachial artery diameter from baseline to the end of the test. Brachial artery internal diameter (ID) was defined as the distance between the intima-lumen interface of the near-wall and lumen-intima interface of the far wall. The two tests were performed on the same day at least $30 \mathrm{~min}$ apart, and the second test 
was not performed until the baseline brachial artery diameter was restored. In both tests, the brachial artery was imaged $\sim 3-4 \mathrm{~cm}$ above the elbow in the longitudinal section on the anterior side of the biceps muscle keeping the angle between the ultrasound beam and the vessel at $90^{\circ}$. The gain was fine-tuned until the lumen-intima interface was clear. Images were recorded for offline measurement by the software Autodesk1 Design Review (13).

Ischemic test was carried out by inflating a pneumatic cuff around the forearm up to $250 \mathrm{mmHg}$ and maintaining inflation for $5 \mathrm{~min}$. Brachial artery ID was recorded at baseline, 1, $2 \mathrm{~min}$, and $3 \mathrm{~min}$ after cuff release.

An exercise test was performed using the handgrip manometer. Participants were instructed on how to perform a maximal voluntary contraction (MVC) and a short bout of isometric handgrip exercise in a $2 \mathrm{~s}$ contraction:3s relaxation ratio with the non-dominant arm. The exercise test consisted of 12 contractions $\min ^{-1}$ for a total of 6 min of handgrip exercise at $30 \%$ intensity of MVC. MVC was evaluated at the beginning of the visit to avoid any interference with vascular tests. The duration and the intensity of the exercise were preliminarily established in healthy volunteers. Six min and $30 \%$ of MVC represented the maximal contraction, ensuring an adequate vasodilation without systemic hemodynamic changes (heart rate and blood pressure) and pain or fatigue.

FMD after ischemia was calculated using the following formula: [(after ischemia ID)-(baseline ID)/(baseline ID)] $\times 100$. The highest FMD among those calculated at 1, 2, and 3 min after cuff deflation was defined as peak FMD. FMD during exercise was expressed as percentage change from baseline and calculated using the following formula: [(1-2-3-4-5-6- min exercise ID)-(baseline ID)/(baseline ID)] ×100.

The reproducibility of the FMD test after ischemia and exercise was calculated in 6 patients with T1D and 5 healthy subjects. Vascular studies were performed on two different days and in the same condition. The coefficient of correlation between the first and the second ischemic test was 0.99 , and between the first and the second exercise test 0.98 .

Statistical analyses: All tests were performed using SPSS 23 for Microsoft (SPSS, Inc., Chicago, IL). Variables not normally distributed were time spent in hypoglycemia, and GV; non-parametric tests or 2Step Rank transformation before applying parametric tests were used. The sample size was calculated using the correlation sample size formula for one group: $N=\left[\left(Z_{a}+Z_{b}\right) / C\right]^{2}+3$, where $Z_{a}=1.96, Z_{b}=0.842$, $a=0.05$ and $b=0.20$. Currently, no data are evaluating the association between glucose metrics and preclinical atherosclerosis. Therefore, the expected correlation between glucose metrics and IMT, FMD in T1D patients we used in the formula was 0.35 and set according to data concerning the association between IMT, FMD, and age or disease duration (12). The total calculated number with an anticipated drop-out $=20 \%$ was 78 .

Parameters were analyzed in T1D patients and patients divided according to the instrument used to monitor blood glucose (SMBG and CGM group) or according to ongoing treatment [continuous subcutaneous insulin infusion (CSII) and multiple daily insulin injection (MDI) group]. TIR, TAR, mean 
daily glucose, and standard deviation (SD) were collected from the diabetes management software. GV was expressed as the coefficient of variation (CV) calculated using the following formula: $\mathrm{CV}=(\mathrm{SD} / \mathrm{mean}$ daily glucose). The $t$-test for paired data and Mann-Whitney $U$ for unpaired data were used to compare continuous variables between T1D and control group and between SMBG and CGM group, and between CSII and MDI group. The Chi-squared test was used to compare the percentage between groups.

Repeated measures ANOVA test was applied to evaluate differences among arterial dilation detected for every min up to 6 min of handgrip exercise in T1D and control group. The one-way ANOVA was used to compare arterial dilation during exercise between SMBG and CGM groups, and between CSII and MDI groups. The simple regression analysis was performed to evaluate the association between mean maximal IMT, FMD (peak FMD and FMD at the $6^{\text {th }}$ min of exercise) and age, disease duration, and glucometric parameters; results have been shown as coefficient of correlation and square of correlation.

\section{Results}

Seventy-eight patients with T1D who met inclusion criteria and 35 healthy control subjects were enrolled. Mean age and male sex prevalence were comparable between T1D and control group. In detail, age (mean \pm SD): T1D $35 \pm 13$ years, control group $31 \pm 13$ years; male sex: T1D 59\%, control group $65 \%$. Mean disease duration and $\mathrm{HbA} 1 \mathrm{c}$ in patients with T1D were respectively $17 \pm 9$ years, and $7.7 \pm 0.9 \%$ ( $61 \mathrm{mmol} / \mathrm{mol})$. Among T1D patients, 29 were monitoring blood glucose by SMBG, and 49 were using CGM. Twenty-five were on CSII and 53 on MDI. No difference in age, disease duration, male sex, and $\mathrm{HbA1c}$ was detected between SMBG and CGM. Patients in MDI were significantly younger then CSII (MDI $31 \pm 10$ vs. CSII $44 \pm 14$ years, $p<0.0001$ ), had a lower disease duration (MDI $15 \pm 8$ vs. CSII $21 \pm 12$ years, $\mathrm{p}<0.01$ ), while male sex prevalence, and $\mathrm{HbA} 1 \mathrm{c}$ were comparable.

Table 1 shows glucose metrics evaluated 2 weeks and 3 months before HbA1c measurement in T1D patients. No statistically significant difference was detected between two-time intervals.

Table 1

Glucose metrics measured over 2-week and 3-month time intervals before HbA1c in T1D patients.

\begin{tabular}{|lll|}
\hline & 2 weeks & 3 months \\
\hline Percent TIR (\%) & $56 \pm 15$ & $55 \pm 13$ \\
\hline Percent TBR (\%) & $9 \pm 11$ & $8 \pm 6$ \\
\hline Percent TAR (\%) & $36 \pm 16$ & $36 \pm 15$ \\
\hline Percent CV (\%) & $41 \pm 11$ & $41 \pm 9$ \\
\hline $\begin{array}{l}\text { TIR: Time In Range; TAR: Time Above range; } \\
\text { Data are expressed as mean } \pm \text { SD. }\end{array}$ & & \\
\hline
\end{tabular}

SMBG users spent more time in hypoglycemia than CGM at both intervals (TBR 2 weeks: SMBG $14 \pm$ 16vs. CGM $5 \pm 5 \%, p<0.001$; TBR 3 months: SMBG $10 \pm 7 v$ s.CGM6 $\pm 4 \%, p<0.03)$, and showed higher CV 
measured over 2 weeks (CV 2 weeks: SMBG $44 \pm 16 v s$. CGM $39 \pm 10, p<0.03$ ). No difference in metrics was detected when patients were divided according to CSII and MDI treatment.

Table 2 shows mean maximal IMT, baseline brachial artery ID, and FMD after ischemia in T1D and control subjects. Baseline brachial artery diameter was comparable between groups. Among T1D, 66 had maximal dilation after $1 \mathrm{~min}, 4$ after $2 \mathrm{~min}$, and 8 did not dilate at all. All healthy subjects had maximal dilation at 1 min. Mean maximal IMT CCA was significantly higher, and FMD significantly lower in T1D.

Table 2

Mean maximal IMT of CCA, baseline brachial artery diameter, and FMD after ischemia in patients with T1D and control subjects.

\begin{tabular}{|lll|}
\hline Variable & T1D & Controls \\
\hline Number & 78 & 35 \\
\hline Mean maximal IMT of CCA $(\mu)$ & $643 \pm 196$ & $536 \pm 111^{\star}$ \\
\hline Baseline brachial artery ID $(\mathrm{mm})$ & $3.4 \pm 0.6$ & $3.4 \pm 0.4$ \\
\hline Percent FMD 1 min $(\%)$ & $7.5 \pm 4.1$ & $10.1 \pm 1.9^{\wedge}$ \\
\hline Percent peak FMD (\%) & $7.5 \pm 4.0$ & $10.1 \pm 1.9^{\wedge}$ \\
\hline $\begin{array}{l}\text { IMT CCA: Intima plus media thickness of common carotid arteries. FMD: Flow Mediated Dilation. } \\
\text { Data are expressed as mean } \pm \text { SD. ANOVA * } \mathrm{p}=0.001 ;{ }^{\wedge} \mathrm{p}=0.0001 .\end{array}$ \\
\hline
\end{tabular}

Mean maximal IMT of CCA and FMD after ischemia were comparable in SMBG and CGM users. Mean maximal IMT of CCA was significantly higher in CSII patients $(784 \pm 246 \mu)$ than MDI $(577 \pm 124 \mu), p<$ 0.0001, while FMD after ischemia was comparable.

FMD was then evaluated during exercise, and the curves representing the percent of dilation measured every min up to 6 min in T1D and control subjects are displayed in Fig. 1. The percentage of dilation progressively and significantly increased in the two groups during exercise. However, FMD was significantly lower in T1D compared with control subjects at every min up to 6 min (ANOVA $p<0.0001$ ). Again, no significant difference was detected between SMBG and CGM groups, while CSII patients showed reduced FMD during exercise compared to MDI. Specifically, FMD during exercise was: 1 min CSII $1.4 \pm 2.3$ vs. MDI $2.2 \pm 2.7 ; 2 \min$ CSII $3.6 \pm 3.3$ vs. MDI $5.6 \pm 3.5 ; 3 \min$ CSII $3.8 \pm 3.2$ vs. MDI $5.9 \pm 3.8$; 4 min CSII $4.6 \pm 3.3$ vs. MDI $6.9 \pm 3.6 ; 5 \min$ CSII $4.9 \pm 3.5$ vs. MDI $6.9 \pm 3.5 ; 6 \min$ CSII $5.0 \pm 3.6$ vs. MDI $7.3 \pm 3.6(p<0.02$ at every $\min )$.

We have evaluated the association between mean maximal IMT, FMD, and glucose metrics (TIR, TBR, TAR, CV), age, disease duration, and HbA1c in patients with T1D (Table 3). Age and disease duration were the only independent variables associated with mean maximal IMT and FMD. 
Table 3

Coefficient of correlation and square of the correlation between independent variables significantly associated with mean maximal IMT of CCA, FMD after ischemia (peak), and FMD at the 6th min of exercise in patients with T1D.

\begin{tabular}{|llll|}
\hline & IMT of CCA $(\mu)$ & Peak FMD (\%) & Exercise FMD 6 min (\%) \\
\hline & $\mathrm{R} / \mathrm{R}^{2}$ & $\mathrm{R} / \mathrm{R}^{2}$ & $\mathrm{R} / \mathrm{R}^{2}$ \\
\hline Age & $0.75 / 0.56^{\star}$ & $-0.26 / 0.10^{\wedge}$ & $-0.29 / 0.10^{\wedge}$ \\
\hline Disease Duration & $0.48 / 0.23^{*}$ & $-0.31 / 0.10^{\wedge}$ & $-0.23 / 0.06 \#$ \\
\hline${ }^{*} \mathrm{p}<0.0001 ;{ }^{\wedge} \mathrm{p}<0.02 ; \# \mathrm{p}<0.05$ & & \\
\hline
\end{tabular}

In detail, for each 10 years of age, mean maximal IMT increased by $110 \mu$; for each 10 years of disease duration, mean maximal IMT increased by $97 \mu$. As well for each 10 years of age, FMD after ischemia (peak) and exercise (6 min) decreased respectively by $1 \%$ or $0.8 \%$; for each 10 years of disease duration, FMD after ischemia (peak) and exercise ( $6 \mathrm{~min}$ ) decreased by $1.3 \%$ and $0.9 \%$, respectively. The lack of association between TIR and other metrics with IMT and FMD was also confirmed in SMBG, CGM, CSII, and MDI groups when analyzed separately.

In control subjects mean maximal IMT was directly associated with age ( $\left.0.63, R^{2} 0.40, p<0.0001\right)$. No association was detected between FMD and age.

\section{Discussion}

The main finding of our study is that the new glucose metrics do not associate with markers of early atherosclerosis, such as common carotid artery IMT and FMD.

Before discussing these results in detail, it is necessary to underline two findings of the present studyfirst, patients with T1D exhibit higher IMT value, and lower vasodilation than healthy control subjects; second, within patients with T1D, with increasing age and years of disease, IMT increases and FMD decreases. These findings confirm similar observations already known in the literature and demonstrate that the data have been correctly collected and are consistent with what is expected (10-15).

The lack of association between glucose-metabolic control and early markers of atherosclerosis is, therefore, disappointing. The possible explanations for this are manifold and are discussed below.

The chosen periods ( 2 weeks, 3 months) may be too short of influencing the measured atherosclerosis markers. The 2-week time was chosen to check whether a proper glucose-metabolic control of even a few days could affect endothelial function. The 3-month time, which more closely reflects glycosylated hemoglobin, was chosen to evaluate the effect of a more extended compensation period on both endothelial function and arterial wall thickness. We cannot rule out that an extended glucose-metabolic control period may have significant effects on the measured vascular parameters. Furthermore, TIR 
indicates the percentage of values between $70-180 \mathrm{mg} / \mathrm{dL}$ overall the day. It does not explicitly identify post-prandial hyperglycemic exposure, that is independently associated with CVD and endothelial function $(26,27)$. The $70-180 \mathrm{mg} / \mathrm{dl}$ range may be too broad, and may not precisely separate patients with proper metabolic compensation from those with less acceptable blood glucose. Another possible explanation is that our measurement systems were not accurate enough to detect small differences, such as those expected in this study. However, the measurement of IMT and FMD was done with very sensitive instruments and following suggested protocols. Measurement of IMT is a well-standardized technique, and despite mean maximal IMT in our T1D patients is below the value considered at high risk for incident cardiovascular events, it is higher than IMT detected in healthy subjects with similar age. Two different techniques have explored endothelial function; the widely used FMD technique with the induction of forearm ischemia stimulating vasodilation and the original one, exercise FMD with the use of standardized handgrip exercise $(28,29)$. Exercise at a submaximal level is a physiological stimulus able, in a short time, to cause vasodilation without systemic cardiovascular adaptations. Therefore, even if both techniques explore the endothelial NO pathway, the vasodilatory response may be earlier dumped during exercise than after ischemia, which is a more intense stimulus. Clinical studies have demonstrated that there is a significant $8-13 \%$ higher cardiovascular risk for each percent point decrease in brachial artery $\operatorname{FMD}(30,31)$. FMD after ischemia was $7.5 \%$ in T1D, and $10.1 \%$ in control subjects; during exercise $6.6 \%$ in T1D, and $9.4 \%$ in controls, a difference higher than $1 \%$. Again this finding is worthy of attention even if obtained from an observational study.

The present study also shows that hypoglycemia and glycemic variability are not associated with FMD and IMT. The relationship between hypoglycemia and CVD is complex and strongly influenced by age, disease duration, glycemic legacy, rate of severe hypoglycemic events, and comorbidities $(32,33)$. However, hypoglycemia does not seem to affect IMT or endothelial function in non-frail patients without longstanding uncontrolled hyperglycemia (34). GV has attracted research in the last years. Again, studies indicate that variability defined as the rate of deviation from the target range in both directions might predict cardiovascular events. An interesting paper has demonstrated how glucose fluctuations may affect endothelial function (35). However, the lack of robust prospective data and the use of different indexes describing GV still limit the interpretation of this parameter.

The present study has several limitations and some strengths. Among the limitations, again, we underline the time range chosen to evaluate glycemic compensation, and the lack of information about fasting and postprandial glycemia. About the first point, we have already explained why the two time periods have been chosen. The evaluation of longer time interval should have taken into account several confounding variables and went beyond the scope of the present study. About the last point, we may argue that more information on fasting and postprandial glucose could have helped to understand if changes in the short term can influence early markers of atherosclerosis. Further studies need to be planned in the future. The strengths of the present study are the number of patients enrolled and the accuracy of the vascular information. 


\section{Conclusion}

Our study demonstrates that new metrics do not help to identify patients with T1D and abnormal morphology and function of extra-coronary arteries, at least in the setting we used in the current research. Different molecules or pathways might be involved in the development of early atherosclerosis in patients with T1D and without long disease duration. Our study reinforces the evidence that the damage of vasculature occurs early in T1D, and all the efforts should be made to reduce the risk of cardiovascular mortality and morbidity.

\section{Abbreviations}

ANOVA

ANalysis Of Variance

CCA

common carotid artery

CGM

Continuous Glucose Monitoring

CSII

Continuous Subcutaneous Insulin Infusion

CV

Coefficient of Variation

CVD

cardiovascular disease

DCCT

Diabetes Control and Complications Trial

DMS

diabetes management system

FMD

Flow Mediated Dilation

FPG

Fasting plasma glucose

GV

Glycemic Variability

$\mathrm{HbA1c}$

Glycated Hemoglobin

ID

Internal Diameter

IMT

Intima-Media Thickness

MDI 
Multiple Daily Insulin injection

MVC

Maximal Voluntary Contraction

NO

Nitric Oxide

SMBG

Self-Monitoring Blood Glucose

T1D

Type 1 diabetes

TAR

Time Above the target Range

TBR

Time Below the target Range

TIR

Time spent In the target Range

\section{Declarations}

Ethics approval and consent to participate: the study was approved by the local Ethical Committee and all participants signed the informed consent;

Consent for publication: all the authors gave their consent for publication;

Availability of data and materials: data will be available whenever requested;

Competing interests: There is no competing of interest

Funding: NA

Authors' contributions: $\mathrm{AC}$ and $\mathrm{Cl}$ study design, vascular procedures, writing manuscript; MP, MM recruitment of participants and data collection; RF, uploading glucose data; AG data analyses and manuscript editing;

Acknowledgements: NA

\section{References}

1. Libby P, Nathan DM, Abraham K, Brunzell JD, Fradkin JE, Haffner SM, Hsueh W, Rewers M, Roberts BT, Savage PJ, Skarlatos S, Wassef M, Rabadan-Diehl C. Report of the national heart, lung, and blood institute-national institute of diabetes and digestive and kidney diseases working group on cardiovascular complications of type 1 diabetes mellitus. Circulation. 2005;111:3489-93. 
2. Giannini C, Mohn A, Chiarelli F, Kelnar CJ. Macrovascular angiopathy in children and adolescents with type 1 diabetes. Diabetes Metabolism Research Reviews. 2011;27:436-60.

3. Rawshani A, Sattar N, Franzen S, Rawshani A, Hattersley AT, Svensson AM, Eliasson B, Gudbjornsdottir S. Excess mortality and cardiovascular disease in type 1 diabetes in relation to age at onset: a nationwide study of 27,195 young adults with diabetes. Lancet. 2018;392:477-86.

4. Vanhoutte PM, Shimokawa H, Feletou M, Tangh EH. Endothelial dysfunction and vascular disease: A thirtieth anniversary update. Acta Physiol. 2017;219:22-96.

5. Lorenz MW, Markus HS, Bots ML, Rosvall M, Sitzer M. Prediction of clinical cardiovascular events with carotid intima-media thickness: a systematic review and meta- analysis. Circulation. 2007;115:459-67.

6. Irace C, De Rosa S, Tripolino C, Ambrosio G, Covello C, Abramo E, Carallo C, Mongiardo A, Spaccarotella C, Torella D, Gnasso A, Indolfi C. Delayed flow-mediated dilation and criticalcoronarystenosis. J Investig Med. 2018;66:10.1136/jim-2017-000644.

7. Inaba Y, Chen JA, Bergmann SR. Prediction of future cardiovascular outcomes by ow-mediated vasodilatation of brachial artery: a meta-analysis. Int J Cardiovasc Imaging. 2010;26:631-40.

8. Yeboah J, Crouse JR, Hsu FC, Burke GL, Herrington DM. Brachial ow-mediated dilation predicts incident cardiovascular events in older adults: The Cardiovascular Health Study. Circulation. 2007;115:2390-97.

9. Xu Y, Arora RC, Hiebert BM, Lerner B, Szwaicer A. McDonald K, Rigatto C, Komenda P, Sood MM, Tangri N. Non-invasive endothelial function testing and the risk of adverse outcomes: a systematic review and meta-analysis. Eur Heart J Cardiovasc Imaging. 2014;15:736-46.

10. Pujia A, Gnasso A, Irace C, Colonna A, Mattioli PL. Common carotid arterial wall thickness in NIDDM subjects. Diabetes Care. 1994;17:130-6.

11. Kawamori R. Asymptomatic hyperglycemia and early atherosclerotic change. Diabetes Res Clin Pract. 1998;40:35-42.

12. Yamasaki Y, Kawamori R, Matsushima H, Nishizawa H, Kodama M, Kubota M, Kajimoto Y, Kamada T. Asymptomatic hyperglycemia is associated with increased intimal plus media thickness of the carotid artery. Diabetologia. 1995;38:585-91.

13. Irace C, Tschakovsky ME, Carallo C, Cortese C, Gnasso A. Endothelial dysfunction or dysfunctions? Identification of three different FMD response in males with type 2 diabetes. Atherosclerosis. 2008;200:439-45.

14. Dogra G, Rich L, Stanton K, Watts GF. Endotheliumdependent and independent vasodilation studies at normoglycaemia in type I diabetes mellitus with and without microalbuminuria. Diabetologia. 2001;44:593-601.

15. Wang P, Xu YY, Lv TT, Guan SY, Li XM, Li XP, Pan HF. Subclinical atherosclerosis in patients with Type 1 diabetes mellitus: a systematic review and meta-analysis. Angiology. 2018.

doi:10.1177/0003319718787366.

Page 12/15 
16. Pillary S, Anderson J, Couper J, Maftei O, Gent R, Pena AS. Children with type 1 diabetes have delayed flow-mediated dilation. Can J Diabetes. 2018;42:276-80.

17. Battelino T, Danne T, Bergenstal RM, Amiel SA, Beck R, Biester T, Bosi E, Buckingham BA, Cefalu WT, Close KL, Cobelli C, Dassau E, DeVries JH, Donaghue KC, Dovc K, Doyle FJ III, Garg S, Grunberger G, Heller S, Heinemann L, Hirsh IB, Hovorka R, Jia W, Kordonouri O, Kovatchev B, Kowalski A, Laffel L, Levine B, Mayorov A, Mathieu C, Murphy HR, Nimri R, Norgaard K, Parkin CG, Renard E, Rodbard D, Saboo B, Schatz D, Stoner K, Urakami T, Weinzimer SA, Phillip M. Clinical targets for Continuous Glucose Monitoring data interpretation: Recommendation from the international consensus on Time in Range. Diabetes Care. 2019;42:1593-603.

18. Beck RW, Bergenstal RM, Cheng P, Kollman C, Carlson AL, Johnson ML, Rodbard D. The relationships between time in range, hyperglycemia metrics and $\mathrm{HbA1c}$. Journal of Diabetes Science Technology. 2019;13:614-26.

19. Vigersky RA, McMahon C. The relationship of hemoglobin A1c to time-in-range in patients with diabetes. Diabetes Technol Ther. 2019;21:81-5.

20. Lu J, Ma X, Zhou J, Mo Y, Ying L, Lu W, Bao Y, Vigersky RA, Jia W. Association of time in range as assessed by continuous glucose monitoring with diabetic retinopathy in type 2 diabetes. Diabetes Care. 2018;41:2370-76.

21. Beck RW, Bergenstal RM, Riddlesworth TD, Kallman C, Li Z, Brown AS, Close KL. Validation of time in range as an outcome measure for diabetes clinical trials. Diabetes Care. 2019;42:400-5.

22. Guo Q, Zang Pu, Shaoying X, Song W, Zhang Z, Liu C, Guo Z, Chen J, Lu B, Gu P, Shao J. Time in range, as a novel metric of glycemic control, is reversely associated with presence of diabetic cardiovascular autonomic neuropathy independent of $\mathrm{HbA} 1$ in Chinese type 2 diabetes. Journal of Diabetes Research. 2020. doi 10.1155/2020/5817074.

23. Irace C, Carallo C, De Franceschi MS, Scicchitano F, Milano M, Tripolino C, Scavelli F, Gnasso A. Human common carotid wall shear stress as a function of age and gender: a 12-year follow-up study. Age. 2012;34:1553-62.

24. Thijssen D, Black M, Pyke KE, Padilla J, Atkinson G, Harris RA, Parker B, Wildansky ME, Tschakovsky $\mathrm{ME}$, Green DJ. Assessment of flow-mediated dilation in humans: a methodological and physiological guideline. Am J Physiol Heart Circ Physiol. 2011;300:2-12.

25. Tremblay JC, Pyke KE. Flow-mediated dilation stimulated by sustained increases in shear stress: a useful tool for assessing endothelial function in humans? Am J Physiol Heart Circ Physiol. 2018;314:508-20.

26. Kawano H, Motoyama T, Hirashima O, Hirai N, Miyao Y, Sakamoto T, Kugiyama K, Ogawa H, Yasue H. Hyperglycemia rapidly suppresses flow-mediated endothelium-dependent vasodilation of brachial artery. J Am Coll Cardiol. 1999;34:146-54.

27. Ceriello A. Hyperglycemia and the vessel wall: the pathophysiological aspects on the atherosclerotic burden in patients with diabetes. Eur J Cardiovasc Prev Rehabil. 2010;17:15-9. 
28. Thijsse DHJ, Bruno RM, van Mil ACCM, Holder SM, Faita F, Greyling A, Zock PL, Taddei S, Deanfield JE, Lusher T, Green DJ, Ghiadoni L. Expert consensus and evidence-based recommendation for the assessment of flow-mediated dilation in humans. Eur Heart J. 2019. doi:10.1093/euheartj/ehz350.

29. Wray DW, Witman MAH, Ives SJ, McDaniel J, Fjeldstad AS, Trinity JD, Conklin JD, Supiano MA, Richardson RS. Progressive handgrip exercise: evidence of nitric oxide-dependent vasodilation and blood flow regulation in humans. Am J Physiol Heart Circ Physiol. 2011;300:H1101-7.

30. Ras RT, Streppel MT, Draijer R, Zock PL. Flow-mediated dilation and cardiovascularrisk prediction: a systematic review with meta-analysis. Int J Cardiol. 2013;168:344-51.

31. Xu Y, Arora RC, Hiebert BM, Lerner B, Szwajcer A, McDonald K, Rigatto C, Komenda P, Sood MM, Tangri N. Non-invasive endothelial function testing and the risk of adverse outcomes: a systematic review and meta-analysis. Eur Heart J Cardiovasc Imaging. 2014;15:736-46.

32. Bonds DE, Miller ME, Bergenstal RM, Buse JB, Byington RP, Cutler JA, Dudi RJ, Ismail-Beigi F, Kimel AR, Hoogwef B, Hoeowitz KR, Savage PJ, Seaquist ER, Simmons DL, Sivitz WI, Speril-Hillen JM, Sweeney ME. The association between symptomatic, severe hypoglycaemia and mortality in type 2 diabetes: retrospective epidemiological analysis of the ACCORD study. BMJ. 2010;340:b4909.

33. Skyler JS, Bergenstal R, Bonow RO, Buse J, Deedwania P, Gale EA, Howard BV, Kirkman MS, Kosiborod M, Reaven P, Sherwin RS. Intensive glycemic control and the prevention of cardiovascular events: implications of the ACCORD, ADVANCE, and VA Diabetes Trials: a position statement of the American Diabetes Association and a scientific statement of the American College of Cardiology Foundation and the American Heart Association. J Am Coll Cardiol. 2009;53:298-304.

34. Irace C, Cutruzzolà A, Carbotti DF, Mastroianni S, Cavallo M, Gnasso A. Hypoglycemia does not affect the progression of preclinical atherosclerosis in subjects with type 2 diabetes. PLoSOne 2019;14:doi:10.1371/journal.pone.0212871.

35. Costantino S, Paneni F, Battista R, Castello L, Capretti G, Chiandotto S, Tanese L, Russo G, Pitocco D, Lanza GA, Volpe M, Lüscher TF, Cosentino F. Impact of glycemic variability on chromatin remodeling, oxidative Stress, and endothelial dysfunction in patients with type 2 diabetes and with target $\mathrm{HbA}_{1 \mathrm{c}}$ levels. Diabetes. 2017;66:2472-82.

\section{Figures}




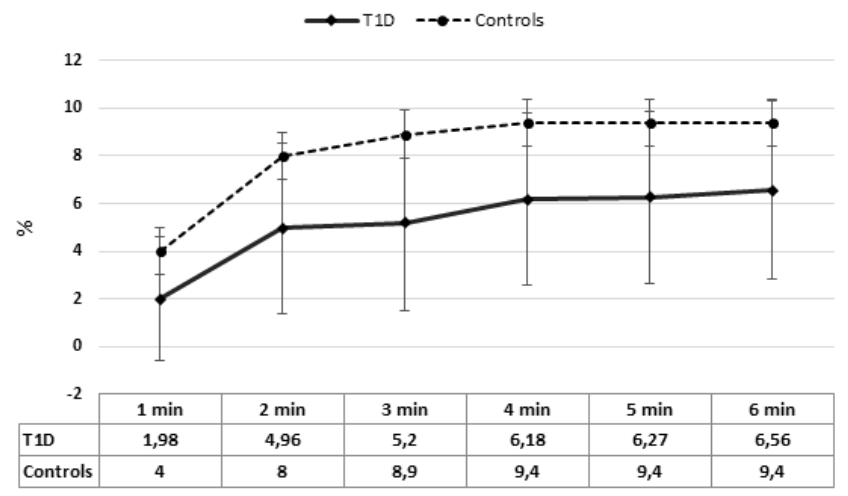

Figure 1

FMD during hand-grip exercise in T1D and control subjects. 